\title{
Molecular characteristics of extended-spectrum $\beta$-lactamase-producing Escherichia coli in Riyadh: emergence of CTX-M-15-producing E. coli ST131
}

\author{
Mohamed H Al-Agamy ${ }^{1,2^{*}}$, Atef M Shibl ${ }^{1,5,6}$, Mohamed M Hafez ${ }^{3,4}$, Mohammad N Al-Ahdal ${ }^{5}$, Ziad A Memish ${ }^{6,7}$ \\ and Harish Khubnani ${ }^{8}$
}

\begin{abstract}
Background: The prevalence of extended-spectrum $\beta$-lactamase-producing Escherichia coli (ESBL-EC) has increased recently. The aim of this study was to further characterise and to assess the occurrence of ESBL-EC in Riyadh, to use pulsed field gel electrophoresis (PFGE) typing to investigate the epidemiology of ESBL-EC and to determine the prevalence of ST131 in ESBL-EC.

Methods: A total of 152 E. coli isolates were collected at a tertiary hospital in Riyadh from September 2010 to June 2011. Genotypic and phenotypic methods were used to characterise ESBLs. PFGE was used to determine genetic relatedness. Detection of ST131 and CTX-M-like ESBLs was performed using real-time PCR.

Results: Of 152 strains, 31 were positive for ESBLs by phenotypic methods. The bla $a_{C T X-M-15}$ gene was highly prevalent (30/31 strains, 96.77\%) among the 31 ESBL-positive E. coli strains. The bla $a_{\mathrm{CTX}-\mathrm{M}-27}$ gene was detected in one strain. Twenty (64.5\%) out of 31 of ESBL-EC were ST131. PFGE revealed 29 different pulsotypes.

Conclusions: Our study documented the high prevalence of ESBLs in E. coli isolates, with CTX-M-15 as the predominant ESBL gene. ST131 clone producing CTX-M-15 has a major presence in our hospital. The high prevalence of CTX-M producers was not due to the spread of a single clone. To the best of our knowledge, this study represents the first report of CTX-M-15 and CTX-M-27 $\beta$-lactamases and the detection of the ST131 clone in Saudi E. coli isolates.
\end{abstract}

Keywords: $\beta$-lactam resistance, Class A $\beta$-lactamases, PFGE, ST131, Saudi Arabia

\section{Background}

Extended-spectrum $\beta$-lactamase-producing Escherichia coli (ESBL-EC) pose a serious threat to the successful treatment of common bacterial infections. Over the past two decades, there has been an increase in the prevalence of ESBL-EC [1]. In addition to TEM and SHV variants of ESBLs, CTX-M enzymes have replaced TEM and SHV in the past several years. CTX-M $\beta$-lactamases have emerged as the predominant ESBL type worldwide [2]. Within the CTX-M family, CTX-M-15 is currently the most widely disseminated CTX-M genotype [2,3]. Recently, several studies have highlighted the worldwide

\footnotetext{
* Correspondence: malagamy@ksu.edu.sa

'Pharmaceutics and Microbiology Department, College of Pharmacy, King Saud University, P.O. Box 2457, Riyadh 11451, Saudi Arabia

${ }^{2}$ Microbiology and Immunology Department, Faculty of Pharmacy, Al-Azhar University, Cairo, Egypt

Full list of author information is available at the end of the article
}

spread of the E. coli ST131 clone [4-6]. The successful dissemination of CTX-M-15 has been considered to be due to the dissemination of genetic elements and the clonal expansion of a pandemic E. coli clone, ST131, with high virulence potential [7]. The E. coli ST131 clone has been reported worldwide; this clone is frequently multidrugresistant and commonly carries CTX-M-15 [6,8].

Previously published studies in Saudi Arabia have sought to determine the prevalence of ESBL-producing isolates, mainly Klebsiella pneumoniae and, to a lesser extent, E. coli, with CTX-M-15 being the most common ESBL [9-17]. However, there is a paucity of studies on the prevalence of ESBL-EC from Saudi Arabia. Until now, no detailed reports characterizing the genotypes of ESBL enzymes or the spread of the ST131 clone in ESBL-EC out of Saudi Arabia have been published. Therefore, this study was performed to investigate the 
molecular epidemiology and genetic characteristics of clinical ESBL-EC isolates obtained from a tertiary hospital in Riyadh, Saudi Arabia.

\section{Materials and methods \\ Bacterial isolates}

One hundred fifty-two non-consecutive, non-duplicate clinical $E$. coli isolates were obtained from inpatients hospitalised at a tertiary hospital in Riyadh, Saudi Arabia. These isolates were collected over a period of 10 months from September 2010 to June 2011. The samples contained 119 isolates from urine, 11 from sputum, 10 from stool, 9 from blood, and 3 from abscesses. Isolates were identified using traditional bacteriological methods and biochemical testing with an API 20E according to the manufacturer's recommendations. The isolates were stored at $-80^{\circ} \mathrm{C}$ in $15 \%$ glycerol (v/v) in tryptic soy broth.

\section{Antimicrobial susceptibility testing}

Antimicrobial susceptibility testing was performed using the disc diffusion method of the Clinical and Laboratory Standards Institute (CLSI) with discs from BBL (Becton Dickinson, Sparks Glencoe, MD, USA). The minimum inhibitory concentration (MIC) for each antibiotic was determined using the dilution and diffusion method on Mueller-Hinton agar with E-test strips (bioMerieux, Marcy L'Etoile, France). The results were interpreted according to the current guidelines of the CLSI [18]. The following antibiotics were tested: amikacin, amoxicillin, amoxicillin-clavulanate, aztreonam, cefepime, cefotaxime, cefoxitin, ceftazidime, ceftazidime/clavulanate, ciprofloxacin, colistin, fosfomycin, gentamicin, imipenem, meropenem, piperacillin, piperacillin-tazobactam, and tigecycline. E. coli ATCC 25922 was used for quality control.

\section{Phenotypic detection of ESBL production}

ESBL production was detected using the 2011 CLSI recommendations for ESBL screening and confirmation tests [18]. The double-disc synergy test and ESBL strip Etests (bioMerieux, Marcy L'Etoile, France) were performed for the detection and confirmation of ESBLs, respectively. For the double-disc synergy test, a ceftazidime disc $(30 \mu \mathrm{g})$ was placed $20 \mathrm{~mm}$ away from a disc containing ceftazidime-clavulanate $(30 / 10 \mu \mathrm{g})$. When the inhibition zone between at least one of the combination discs and its corresponding single antibiotic disc differed by $\geq 5 \mathrm{~mm}$, the strain was identified as an ESBL producer. For the E-test, an ESBL strip containing ceftazidime and ceftazidime-clavulanate was used to determine the MIC ratio according to the manufacturer's instructions. E. coli ATCC 25922 (negative control) and K. pneumoniae ATCC 700603 (positive control) were used as reference strains.

\section{PCR of $\beta$-lactamases}

Polymerase chain reaction (PCR) and sequence analyses were conducted to determine the gene responsible for the ESBL phenotype in the ESBL producers. PCR for $b l a_{\mathrm{TEM}}, b l a_{\mathrm{SHV}}$, and $b l a_{\mathrm{CTX}-\mathrm{M}}$ genes were conducted using PCR primers and conditions that have been previously described [19]. The five CTX-M subgroups (CTXM-1, CTX-M-2, CTX-M-8, CTX-M-9, and CTX-M-25) were amplified using PCR primers and procedures as previously described [20].

Detection of $b l a_{\mathrm{CTX}-\mathrm{M}-15}$-like genes was performed using real-time PCR according to previously published methods [6]. Primers were used to amplify a 49-bp region that is conserved amongst group 1 CTX-M genes as follows: MC-3-15 F (5'-TGG GGG ATA AAA CCG GCA G-3') and MC-3-15R (5'-GCG ATA TCG TTG GTG GTG $\left.\mathrm{C}-3^{\prime}\right)$. Briefly, a $25-\mu \mathrm{L}$ reaction mixture contained final concentrations of $1 \times$ SYBR Green PCR Master Mix buffer (Applied Biosystems, CA, USA), $0.3 \mu \mathrm{M}$ of each forward and reverse primers, $100 \mathrm{ng}$ of DNA, and RNase/DNase-free water. The reaction was performed in an ABI 96-Well Optical Reaction Plate. The thermal cycling conditions were: initial denaturation at $95^{\circ} \mathrm{C}$ for $10 \mathrm{~min}$, followed by 25 cycles at $95^{\circ} \mathrm{C}$ for $15 \mathrm{sec}$ and $68^{\circ} \mathrm{C}$ for $45 \mathrm{sec}$. Following amplification, melting curve analysis was performed by heating the PCR product from $55^{\circ} \mathrm{C}$ to $95^{\circ} \mathrm{C}$ with a ramp rate of $0.05^{\circ} \mathrm{C} / \mathrm{s}$ to verify the presence of a specific product according to its specific melting temperature. The results were analysed using the melting curve analysis software from Applied Biosystems.

\section{DNA sequencing}

Automatic sequencing was performed on both strands of all PCR products using the ABI Prism 3700 DNA Sequencer (Applied Biosystems, Foster City, CA). The types of $\beta$-lactamase genes were identified by comparison with the sequences in the database of G. Jacoby and K. Bush (http://www.lahey.org/Studies/) and the sequences in GenBank (http://blast.ncbi.nlm.nih.gov/Blast.cgi).

\section{Pulsed-field gel electrophoresis}

Pulsed-field gel electrophoresis (PFGE) of chromosomal DNA digested with XbaI (New England BioLabs, MA, USA) was performed according to a standard protocol [21]. Electrophoresis of the prepared samples was performed on the CHEF-DRIII system (Bio-Rad Laboratories) with 3 litres of $0.5 \mathrm{X}$ TBE running buffer. The gels were run with an initial switch time of $2.2 \mathrm{sec}$, a final switch time of $54.2 \mathrm{sec}$, a run time of 22 hours, an angle of $120^{\circ}$, a gradient of $6.0 \mathrm{~V} / \mathrm{cm}$, and a temperature of $14^{\circ} \mathrm{C}$. Lambda ladder (New England BioLabs) was used as a molecular weight marker. Each gel was stained with $1 \mu \mathrm{g} / \mathrm{ml}$ ethidium bromide for 30 minutes and destained with distilled water for 30 minutes. The gels were photographed 
under UV transillumination. DNA fingerprints were analysed using BioNumerics Software (Applied Maths, Keistraat sint-Martens-laten, Belgium). Cluster analysis was performed based on the Dice coefficient with a $1.8 \%$ band tolerance and an optimisation of $4 \%$ for comparisons of DNA profiles. A similarity coefficient of $80 \%$ was applied to the generated dendrogram, which corresponds to the criteria of Tenover et al. [22].

\section{Detection of the ST131 clone}

ST131 clone detection was performed using real-time PCR according to previously published methods [6]. The following primers were used to detect the ST131clone: ST131TF (5'-GGT GCT CCA GCA GGT G-3') with ST131TR (5'-TGG GCG AAT GTC TGC-3'), and ST131AF (5'-GGC AAT CCA ATA TGA CCC-3') with ST131AR (5'-ACC TGG CGA AAT TTT TCG-3'). The thermal cycling conditions for the ST131 assays included an initial denaturation at $95^{\circ} \mathrm{C}$ for $10 \mathrm{~min}$ followed by 40 cycles at $95^{\circ} \mathrm{C}$ for $15 \mathrm{sec}$ and $60^{\circ} \mathrm{C}$ for $1 \mathrm{~min}$. Reaction conditions and melt curve analyses were carried out as described above.

\section{Results}

Antimicrobial susceptibility and ESBL prevalence

Antimicrobial susceptibility testing results are shown in Table 1. Imipenem, meropenem, colistin, tigecycline, and fosfomycin were the most active agents (susceptibility: $100 \%)$. Out of the 152 strains, $50 \%$ were resistant to

Table 1 Resistance rates for clinical Escherichia coli

\begin{tabular}{|c|c|c|c|}
\hline \multirow[t]{2}{*}{ Antimicrobial agents } & \multicolumn{3}{|c|}{ Number (\%) of resistant isolates } \\
\hline & $\begin{array}{c}\text { ESBL } \\
(n=31)\end{array}$ & $\begin{array}{l}\text { Non-ESBL } \\
(n=121)\end{array}$ & $\begin{array}{c}\text { Total } \\
(n=152)\end{array}$ \\
\hline Amoxicillin & $31(100)$ & $121(100)$ & $152(100)$ \\
\hline Amoxicillin/clavulanic acid & $31(100)$ & $115(95)$ & $146(96)$ \\
\hline Piperacillin & $31(100)$ & $99(81.81)$ & $130(85.52)$ \\
\hline Piperacillin/tazobactam & $24(77.44)$ & $52(42.97)$ & $76(50)$ \\
\hline Cefoxitin & $22(70.3)$ & $87(71.1)$ & $109(71.7)$ \\
\hline Ceftazidime & $31(100)$ & $2(1.65)$ & $33(21.71)$ \\
\hline Cefotaxime & $31(100)$ & $3(2.47)$ & $34(22.36)$ \\
\hline Cefepime & $31(100)$ & $2(1.65)$ & $33(21.71)$ \\
\hline Aztreonam & $31(100)$ & $2(1.65)$ & $33(21.71)$ \\
\hline Imipenem & $0(0.0)$ & $0(0.0)$ & $0(0.0)$ \\
\hline Meropenem & $0(0.0)$ & $0(0.0)$ & $0(0.0)$ \\
\hline Ciprofloxacin & $30(96.77)$ & $49(40.5)$ & $79(51.97)$ \\
\hline Gentamicin & $26(83.87)$ & $37(30.58)$ & $63(41.44)$ \\
\hline Amikacin & $17(54.38)$ & $25(20.66)$ & $42(27.63)$ \\
\hline Colistin & $0(0.0)$ & $0(0.0)$ & $0(0.0)$ \\
\hline Tigecycline & $0(0.0)$ & $0(0.0)$ & $0(0.0)$ \\
\hline Fosfomycin & $0(0.0)$ & $0(0.0)$ & $0(0.0)$ \\
\hline
\end{tabular}

piperacillin/tazobactam, $51.97 \%$ to ciprofloxacin, and $41.44 \%$ to gentamicin. The resistance rates for cefotaxime, aztreonam, cefepime, and amikacin were $22.36 \%, 21.71 \%$, $21.71 \%$, and $27.63 \%$, respectively. Over $96 \%, 85.5 \%$ and $71.7 \%$ of the isolates were non-susceptible to amoxicillin/ clavulanic acid, piperacillin and cefoxitin, respectively.

Of the $152 \mathrm{E}$. coli isolates, 31 (20.4\%) were confirmed to be ESBL producers. Of the 119 uropathogenic E. coli isolates, 11 isolates were from sputum, 10 were from stool, 9 were from blood, and three were from abscesses; 24 (20.16\%), 2 (18.2\%), 1 (10\%), 3 (33.33\%), and 1 (50\%) produced ESBLs, respectively.

The susceptibility and MIC data for the ESBL-EC are summarised in Tables 1 and 2. Of all 31 isolates identified as ESBL producers, 30 isolates (96.77\%) were resistant to ciprofloxacin, $17(54.38 \%)$ to amikacin, $26(83.87 \%)$ to gentamicin, $24(77.44 \%)$ to piperacillin-tazobactam and 22 (70.3\%) to cefoxitin. The tested strains were all susceptible to imipenem, meropenem, colistin, tigecyclin and fosfomycin, and all of them were non-susceptible to amoxicillin, amoxicillin/clavulanic acid, piperacillin, ceftazidime, cefotaxime, cefepime, and aztreonam.

\section{$\beta$-lactamases of ESBL-producing $E$. coli}

Of the $31 \mathrm{E}$. coli isolates with ESBL phenotypes, all tested positive for ESBL production. All ESBL types belonged to the CTX-M family; TEM- or SHV-type ESBL genes were not detected. Of the 31 ESBL-EC isolates, 30 (96.77\%) produced CTX-M-15, and one (3.23\%) produced CTX-M27, a variant of CTX-M-14. Another $\beta$-lactamase, TEM-1, was concomitantly produced by $100 \%$ of all ESBL-EC isolates (Table 2).

\section{Pulsed-field gel electrophoresis}

PFGE was performed on 31 ESBL-EC isolates. Using a $>80 \%$ similarity cut-off point, PFGE analysis showed genetic heterogeneity in the CTX-M-producing E. coli isolates, revealing 29 distinctive pulsotypes (Figure 1). Twentyseven of 29 pulsotypes comprised $87.1 \%$ of the isolates. Each pulsotype contained one strain, except for two pulsotypes, A1 and L1, which contained two strains each. Two pulsotypes represented $12.9 \%$ of all studied ESBL-EC isolates. The results of PFGE revealed that there were no outbreaks or spreading of one single pulsotype in the hospital.

\section{Prevalence of ST131 in ESBL-EC}

Twenty (64.5\%) of 31 ESBL-EC isolates were identified as the ST131 clone. The ST131 ESBL-ES isolates belonged to 18 different pulsotypes. All ST131 isolates produced CTX-M-15. Sixteen (66.6) of 24 uropathogenic ESBL-EC isolates were ST131, while two (66.6\%) of three blood isolates were ST131, one (50\%) of two ESBL-EC isolated from sputum was ST131, and one (100\%) ESBLEC isolated from a wound was ST131. 
Table 2 Clinical specimens, MICs, PFGE, ST131 positivity, and $\beta$-lactamase genes among ESBL-producing E. coli isolates

\begin{tabular}{|c|c|c|c|c|c|c|c|c|c|c|c|c|c|c|c|c|c|c|c|c|c|c|}
\hline \multirow[t]{2}{*}{ No } & \multirow[t]{2}{*}{ Isolate } & \multirow[t]{2}{*}{ Specimen } & \multicolumn{17}{|c|}{ MIC $(\mu \mathrm{g} / \mathrm{ml})$} & \multirow[t]{2}{*}{ Pulsotype } & \multirow[t]{2}{*}{ ST131 } & \multirow[t]{2}{*}{$\beta$-lactamases } \\
\hline & & & AMC & PIP & PTZ & CT & FOX & CAZ & $\mathrm{CAZ} / \mathrm{C}$ & FP & IP & MER & ATM & GM & AMK & CIP & TIG & COL & FOS & & & \\
\hline 1 & EC22 & Urine & $>256$ & 192 & 32 & $>256$ & 2 & 48 & 32 & $>256$ & $\leq 0.25$ & $\leq 0.25$ & $>256$ & $>256$ & 16 & $>32$ & $\leq 0.25$ & $\leq 0.5$ & $\leq 0.25$ & $\mathrm{~A} 1$ & + & TEM-1 + CTX-M-15 \\
\hline 2 & EC30 & Urine & $>256$ & $>256$ & $>64$ & $>256$ & 0.5 & 64 & 32 & $>256$ & $\leq 0.25$ & $\leq 0.25$ & $>256$ & $>256$ & 32 & $>32$ & $\leq 0.25$ & $\leq 0.5$ & $\leq 0.25$ & $\mathrm{~A} 1$ & + & TEM-1 + CTX-M-15 \\
\hline 3 & EC11 & Urine & $>256$ & 192 & 48 & $>256$ & 0.5 & 48 & $>32$ & $>256$ & $\leq 0.25$ & $\leq 0.25$ & $>256$ & $>256$ & 32 & $>32$ & $\leq 0.25$ & $\leq 0.5$ & $\leq 0.25$ & $\mathrm{~A} 2$ & + & TEM-1 + CTX-M-15 \\
\hline 4 & EC27 & Urine & 192 & 128 & 6 & $>256$ & 16 & $>256$ & $>32$ & $>256$ & $\leq 0.25$ & $\leq 0.25$ & 192 & $>256$ & 6 & 24 & $\leq 0.25$ & $\leq 0.5$ & $\leq 0.25$ & B & + & TEM-1 + CTX-M-15 \\
\hline 5 & EC8 & Urine & $>256$ & $>256$ & 8 & $>256$ & 12 & $>256$ & 16 & $>256$ & $\leq 0.25$ & $\leq 0.25$ & $>256$ & $>256$ & $>256$ & $>32$ & $\leq 0.25$ & $\leq 0.5$ & $\leq 0.25$ & C & - & TEM-1 + CTX-M-15 \\
\hline 6 & EC10 & Urine & $>256$ & $>256$ & $>64$ & $>256$ & 0.25 & $>256$ & $>32$ & $>256$ & $\leq 0.25$ & 0.5 & $>256$ & $>256$ & 6 & $>32$ & $\leq 0.25$ & $\leq 0.5$ & $\leq 0.25$ & D & + & TEM-1 + CTX-M-15 \\
\hline 7 & EC1 & Urine & $>256$ & $>256$ & 8 & $>256$ & 32 & $>256$ & 16 & $>256$ & $\leq 0.25$ & $\leq 0.25$ & $>256$ & $>256$ & 16 & $>32$ & $\leq 0.25$ & $\leq 0.5$ & $\leq 0.25$ & E1 & - & TEM-1 + CTX-M-15 \\
\hline 8 & EC6 & Urine & $>256$ & $>256$ & 12 & $>256$ & 24 & $>256$ & 8 & $>256$ & $\leq 0.25$ & $\leq 0.25$ & $>256$ & $>256$ & 2 & $>32$ & $\leq 0.25$ & $\leq 0.5$ & $\leq 0.25$ & E2 & + & TEM-1 + CTX-M-15 \\
\hline 9 & EC12 & Blood & $>256$ & $>256$ & 12 & $>256$ & 32 & $>256$ & 8 & $>256$ & $\leq 0.25$ & $\leq 0.25$ & $>256$ & 64 & 4 & 4 & $\leq 0.25$ & $\leq 0.5$ & $\leq 0.25$ & E3 & + & TEM-1 + CTX-M-15 \\
\hline 10 & EC9 & Sputum & $>256$ & $>256$ & $>64$ & $>256$ & 32 & $>256$ & 8 & $>256$ & $\leq 0.25$ & $\leq 0.25$ & 96 & $>256$ & 6 & $>32$ & $\leq 0.25$ & $\leq 0.5$ & $\leq 0.25$ & $\mathrm{~F}$ & - & TEM-1 + CTX-M-15 \\
\hline 11 & EC31 & Urine & $>256$ & $>256$ & $>64$ & $>256$ & 16 & $>256$ & 8 & $>256$ & $\leq 0.25$ & $\leq 0.25$ & $>256$ & $>256$ & 4 & 2 & $\leq 0.25$ & $\leq 0.5$ & $\leq 0.25$ & G & + & TEM-1 + CTX-M-15 \\
\hline 12 & EC14 & Urine & $>256$ & $>256$ & $>64$ & $>256$ & 48 & $>256$ & 32 & $>256$ & $\leq 0.25$ & 1 & $>256$ & $>256$ & 8 & $>32$ & $\leq 0.25$ & $\leq 0.5$ & $\leq 0.25$ & $\mathrm{H}$ & + & TEM-1 + CTX-M-15 \\
\hline 13 & EC5 & Urine & $>256$ & $>256$ & $>64$ & $>256$ & 16 & $>256$ & 32 & $>256$ & $\leq 0.25$ & $\leq 0.25$ & $>256$ & $>256$ & 2 & $>32$ & $\leq 0.25$ & $\leq 0.5$ & $\leq 0.25$ & 11 & + & TEM-1 + CTX-M-15 \\
\hline 14 & EC32 & Urine & $>256$ & $>256$ & $>64$ & $>256$ & 24 & 128 & 16 & $>256$ & $\leq 0.25$ & $\leq 0.25$ & $>256$ & 96 & 2 & $>32$ & $\leq 0.25$ & $\leq 0.5$ & $\leq 0.25$ & 12 & + & TEM-1 + CTX-M-15 \\
\hline 15 & EC18 & Blood & $>256$ & $>256$ & $>64$ & 4 & 32 & 16 & 2 & 24 & $\leq 0.25$ & $\leq 0.25$ & 48 & $>256$ & 16 & $>32$ & $\leq 0.25$ & $\leq 0.5$ & $\leq 0.25$ & J1 & - & TEM-1 + CTX-M-15 \\
\hline 16 & EC20 & Sputum & $>256$ & $>256$ & $>64$ & $>256$ & 32 & $>256$ & $>32$ & $>256$ & $\leq 0.25$ & $\leq 0.25$ & $>256$ & $>256$ & 48 & $>32$ & $\leq 0.25$ & $\leq 0.5$ & $\leq 0.25$ & $J 2$ & + & TEM-1 + CTX-M-15 \\
\hline 17 & EC2 & Urine & $>256$ & $>256$ & $>64$ & $>256$ & 16 & 96 & 8 & $>256$ & $\leq 0.25$ & $\leq 0.25$ & $>256$ & $>256$ & 4 & $>32$ & $\leq 0.25$ & $\leq 0.5$ & $\leq 0.25$ & $J 3$ & + & TEM-1 + CTX-M-15 \\
\hline 18 & EC3 & Urine & $>256$ & $>256$ & $>64$ & $>256$ & 32 & 24 & 8 & $>256$ & $\leq 0.25$ & $\leq 0.25$ & $>256$ & $>256$ & 8 & $>32$ & $\leq 0.25$ & $\leq 0.5$ & $\leq 0.25$ & $J 4$ & - & TEM-1 + CTX-M-15 \\
\hline 19 & EC13 & Urine & $>256$ & $>256$ & $>64$ & $>256$ & 32 & $>256$ & 32 & $>256$ & $\leq 0.25$ & $\leq 0.25$ & $>256$ & $>256$ & 32 & 8 & $\leq 0.25$ & $\leq 0.5$ & $\leq 0.25$ & K1 & - & TEM-1 + CTX-M-15 \\
\hline 20 & EC33 & Urine & $>256$ & $>256$ & $>64$ & $>256$ & 96 & $>256$ & 16 & $>256$ & $\leq 0.25$ & $\leq 0.25$ & $>256$ & 192 & 64 & 12 & $\leq 0.25$ & $\leq 0.5$ & $\leq 0.25$ & K2 & + & TEM-1 + CTX-M-15 \\
\hline 21 & EC16 & Urine & $>256$ & $>256$ & $>64$ & $>256$ & 24 & $>256$ & 8 & $>256$ & $\leq 0.25$ & $\leq 0.25$ & $>256$ & $>256$ & 64 & 8 & $\leq 0.25$ & $\leq 0.5$ & $\leq 0.25$ & K3 & - & TEM-1 + CTX-M-15 \\
\hline 22 & EC4 & Urine & $>256$ & $>256$ & $>64$ & $>256$ & 0.25 & 192 & 16 & $>256$ & 0.5 & 1.5 & $>256$ & 4 & 2 & $>32$ & $\leq 0.25$ & $\leq 0.5$ & $\leq 0.25$ & L1 & + & TEM-1 + CTX-M-15 \\
\hline 23 & EC15 & Urine & $>256$ & $>256$ & $>64$ & $>256$ & 0.5 & $>256$ & 18 & $>256$ & $\leq 0.25$ & $\leq 0.25$ & $>256$ & 4 & 0.5 & $>32$ & $\leq 0.25$ & $\leq 0.5$ & $\leq 0.25$ & L1 & + & TEM-1 + CTX-M-15 \\
\hline 24 & EC25 & Urine & $>256$ & $>256$ & $>64$ & $>256$ & 0.25 & 12 & 32 & $>256$ & $\leq 0.25$ & $\leq 0.25$ & $>256$ & 3 & 2 & $>32$ & $\leq 0.25$ & $\leq 0.5$ & $\leq 0.25$ & L2 & - & TEM-1 + CTX-M-15 \\
\hline 25 & EC26 & Urine & $>256$ & $>256$ & $>64$ & $>256$ & 192 & $>256$ & $>32$ & $>256$ & $\leq 0.25$ & $\leq 0.25$ & $>256$ & $>256$ & 2 & $>32$ & $\leq 0.25$ & $\leq 0.5$ & $\leq 0.25$ & M1 & + & TEM-1 + CTX-M-15 \\
\hline 26 & EC29 & Wound swab & $>256$ & $>256$ & $>64$ & $>256$ & 0.25 & $>256$ & 8 & $>256$ & $\leq 0.25$ & $\leq 0.25$ & $>256$ & $>256$ & 6 & $>32$ & $\leq 0.25$ & $\leq 0.5$ & $\leq 0.25$ & M2 & + & TEM-1 + CTX-M-15 \\
\hline 27 & EC7 & Blood & $>256$ & $>256$ & $>64$ & $>256$ & 0.25 & $>256$ & 8 & $>256$ & $\leq 0.25$ & $\leq 0.25$ & $>256$ & 2 & 96 & 1 & $\leq 0.25$ & $\leq 0.5$ & $\leq 0.25$ & N & - & TEM-1 + CTX-M-27 \\
\hline 28 & EC19 & Stool & $>256$ & $>256$ & $>64$ & $>256$ & 32 & 3 & 32 & $>256$ & $\leq 0.25$ & $\leq 0.25$ & $>256$ & $>256$ & 128 & $>32$ & $\leq 0.25$ & $\leq 0.5$ & $\leq 0.25$ & O & - & TEM-1 + CTX-M-15 \\
\hline 29 & EC21 & Urine & 128 & 64 & 8 & $>256$ & 128 & 96 & 32 & $>256$ & $\leq 0.25$ & $\leq 0.25$ & $>256$ & $>256$ & $>256$ & $>32$ & $\leq 0.25$ & $\leq 0.5$ & $\leq 0.25$ & $P$ & + & TEM-1 + CTX-M-15 \\
\hline 30 & EC23 & Urine & $>256$ & 192 & 32 & $>256$ & 48 & 24 & 32 & $>256$ & $\leq 0.25$ & $\leq 0.25$ & $>256$ & $>256$ & 128 & $>32$ & $\leq 0.25$ & $\leq 0.5$ & $\leq 0.25$ & Q & - & TEM-1 + CTX-M-15 \\
\hline 31 & EC28 & Urine & $>256$ & $>256$ & 8 & $>256$ & 32 & 4 & 16 & $>256$ & $\leq 0.25$ & $\leq 0.25$ & $>256$ & 4 & 2 & $>32$ & $\leq 0.25$ & $\leq 0.5$ & $\leq 0.25$ & $\mathrm{R}$ & + & TEM-1 + CTX-M-15 \\
\hline
\end{tabular}

Abbreviations: AMK Amikacin, AMC Amoxicillin/Clavulanic acid, AZT Aztreonam, FP Cefepime, FOX Cefoxitin, CAZ Ceftazidime, CT Cefotaxime, CIP Ciprofloxacin, Clav Clavulanic acid, COL Colistin, GM Gentamicin, IP Imipenem, MER Meropenem, PIP Piperacillin, PTZ Piperacillin/tazobactam, TIG Tigecycline, FOS Fosphomycin. 


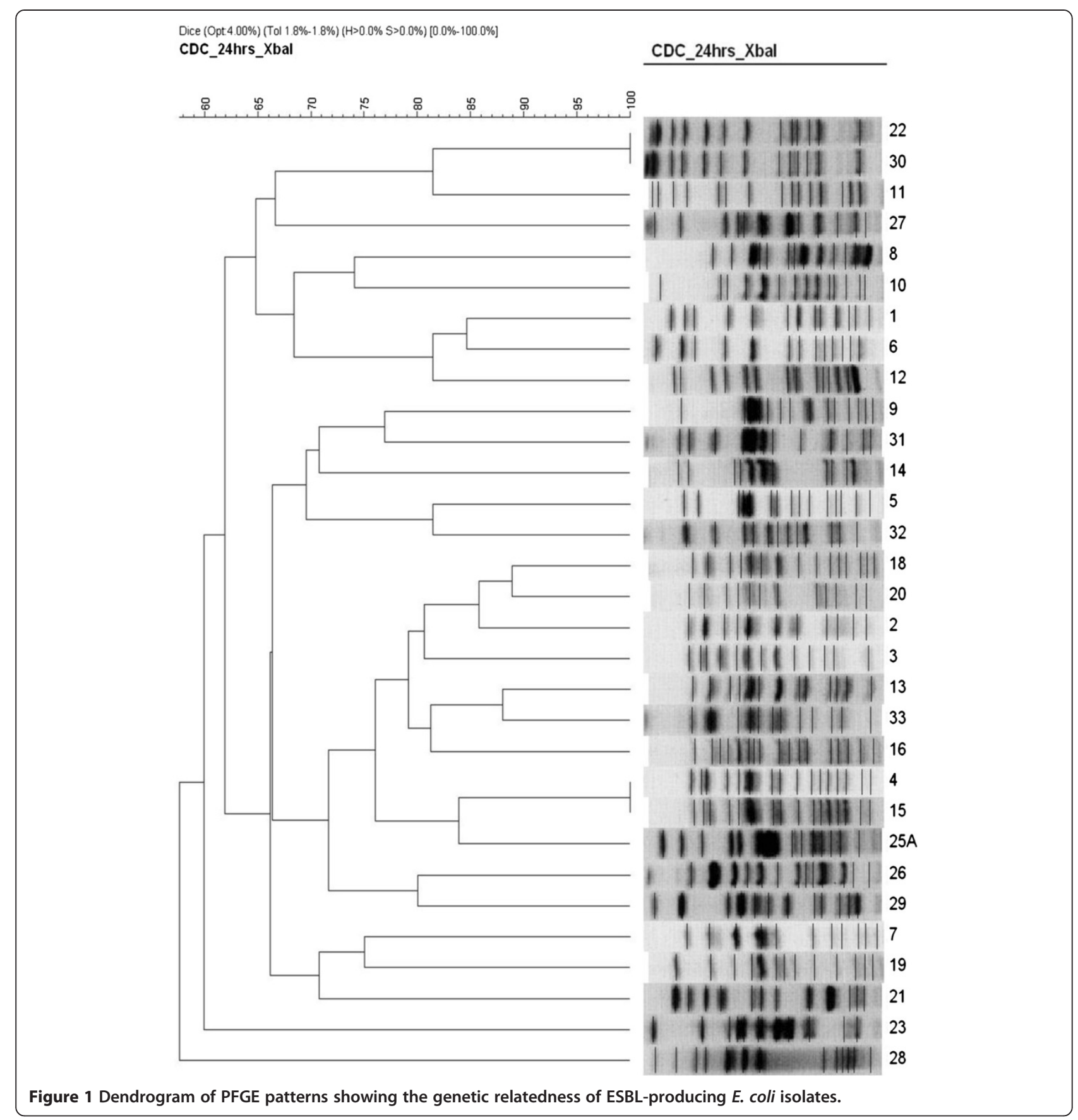

\section{Discussion}

The prevalence of ESBLs among clinical isolates, especially $E$. coli and $K$. pneumoniae, has increased significantly over the past two decades [3]. Despite this increase in ESBLs worldwide, there is a paucity of local reports on the prevalence of ESBL-EC [14-16]. Bindayna et al. [17] reported a high level of bla $a_{\mathrm{CTX}-\mathrm{M} \text {-positive }}$ ESBL-EC; however, they did not determine the type of CTX-M enzymes present. This study is the first in Saudi Arabia to specifically determine the CTX-M-types of ESBLs, to determine strain typing and to determine the spread of the ST131 clone among ESBL-EC isolates sampled from inpatients.

A total of $152 \mathrm{E}$. coli clinical isolates collected from a hospitalised patient population over a 10-month time period from September 2010 to June 2011 were evaluated for the production of ESBL enzymes. The results revealed that $31(20.4 \%)$ of $152 \mathrm{E}$. coli isolates produced ESBL. This study demonstrated an increasing prevalence of ESBL-EC. In previous local studies, the prevalence rates of ESBL-EC were $6.5 \%$ and $10.3 \%$ in 2002 and 2004, respectively [14], and the prevalence rates were 
$15.7 \%$ and $4.8 \%$ from inpatients and outpatients, respectively [15]. Recently, the prevalence of ESBL-EC in uropathogenic E. coli was 33.3\% [16].

Currently, CTX-M enzymes are replacing SHV and TEM enzymes as the prevalent ESBL type [2]. In this study, all ESBL-EC produced the ESBL CTX-M concomitant with the narrow-spectrum $\beta$-lactamase TEM-1. None of the isolates produced SHV $\beta$-lactamase. From our study, we documented that CTX-M enzymes are the dominant ESBLs in Saudi Arabia. CTX-M-15-producing E. coli have spread worldwide [1]. Our results revealed that CTX-M-type enzymes accounted for $100 \%$ of all ESBLs. These results reflect the global trend toward a pandemic spread of CTX-M-type ESBLs in E. coli. While the CTX-M-1 group was predominant, the CTX-M-9 group was rarely reported in Saudi Arabia: within the CTX-M family, CTX-M-1- and CTX-M-9-like genes had prevalence rates of $96.77 \%$ and $3.23 \%$, respectively. DNA sequencing revealed that CTX-M-15 is the sole enzyme with a CTX-M-1-like gene, while CTX-M-27, a variant of CTX-M-14, is the sole enzyme with a CTX-M-9-like gene. This is the first study to describe CTX-M-15 and CTX-M-27 enzymes among ESBL-EC isolates in Saudi Arabia and to document a high prevalence of CTX-M15. In previous local studies, two groups of enzymes; CTX-M-1- and CTXM-9-like genes, were identified in CTX-M-positive $K$. pneumoniae isolates, with prevalence rates of $60 \%$ and $40 \%$, respectively [10]. CTX-M-15 and CTX-M-27 enzymes hydrolyse cefotaxime and ceftazidime. CTX-M-27 confers stronger resistance to ceftazidime than CTX-M-14 [23]. Strikingly, we identified one ESBL-EC isolate producing CTX-M-27 in Saudi Arabia for the first time.

E. coli ST131 is a worldwide pandemic clone that causes antimicrobial-resistant infections. The pandemic spread of CTX-M-15 ESBL-EC was identified in 2008 on three continents $[7,24]$. Since then, the worldwide prevalence of ST131 harbouring a broad range of virulence and resistance genes on a transferable plasmid has been confirmed [7]. E. coli ST131 can harbour a variety of $\beta$ lactamase genes; most often, these include CTX-M, and, less frequently, TEM, SHV, and CMY. The E. coli ST131 clone has been reported worldwide; this clone is frequently multidrug-resistant, commonly carries CTX-M15 [6,8]. CTX-M-15-producing E. coli ST131 has not yet been reported in Saudi Arabia. A particularly interesting result of this study is the finding that ST131 was preva-

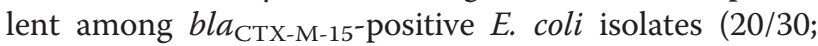
66.66\%) in Saudi Arabia. However, the CTX-M-27-positive isolates were not ST131. Our results suggested that ST131-CTX-M-15-ESBL-EC strains have circulated in Saudi Arabia. A high prevalence of the clone ( 30\%-60\%) has been identified amongst fluoroquinolone-resistant $E$. coli. ST131 comprised 64\% of community-acquired and
$84 \%$ of hospital-acquired cefpodoxime-resistant E. coli infections [25]. ST131 comprised 53\% and 30\% of CTX-M ESBL-producing E. coli in Chicago and Pittsburgh, respectively [26,27]. In Indonesia, $36.8 \%$ of CTX-M-15-positive $E$. coli belonged to the O25b-ST131 lineage [28]. A high prevalence of the CTX-M-15-producing O25bST131 E. coli clone in Bulgaria has been reported [29], as have high proportions of ESBL-producing E. coli ST131 isolates from India, Pakistan, Iran, and Lebanon [30]. In early studies, ST131 clones producing CTX-M-15 had the propensity to cause community-onset infections, especially urinary tract infections [30-32]. However, this clone has also been recently identified in isolates recovered from health care settings, such as hospitals and nursing homes $[33,34]$. There was a high proportion of ST131 among CTX-M-15 producing isolates.

Limitations of this study include that it was performed at a single institution in Saudi Arabia and that a low number of isolates were studied. Furthermore, this study was conducted on hospitalised patients, which does not reflect the epidemiological changes in the Saudi community. Thus, the study results may not reflect the epidemiology of different geographic areas within the kingdom. Despite these limitations, to our knowledge, this is the first study describing the genetic characteristics of ESBL-EC in Saudi Arabia. This study demonstrates a much higher overall prevalence of ESBL-EC than has been previously reported in Saudi Arabia, with CTX-M-type enzymes being the predominant ESBLs. In addition, the study results provide evidence for the dominance of CTXM-15-producing isolates in Saudi Arabia [9,10,12,13,17] and that the predominance of ST131 is likely responsible for the clonal expansion of ESBL-EC in Saudi Arabia, conclusions that are consistent with the changing epidemiology of ESBL-EC worldwide.

\section{Competing interests}

The authors declare that they have no competing interests.

\section{Authors' contributions}

MHA conceived and designed the study, carried out the antibiotic sensitivity, phenotypic detection of ESBL, molecular genetic studies, participated in the sequence alignment and drafted the manuscript. AMS carried out the PFGE and participated in phenotypic detection of ESBL and drafted manuscript. $M M H$ carried out the real-time PCR for detection of ST131. MNA participated in the PFGE. ZAM participated in the design of the study. HK collected the isolates and the clinical data. All authors read and approved the final manuscript.

\section{Acknowledgments}

The authors extend their appreciation to the Deanship of Scientific Research at King Saud University for funding the work through research group project no. RGP-VPP-038.

\section{Author details}

'Pharmaceutics and Microbiology Department, College of Pharmacy, King Saud University, P.O. Box 2457, Riyadh 11451, Saudi Arabia. ${ }^{2}$ Microbiology and Immunology Department, Faculty of Pharmacy, Al-Azhar University, Cairo, Egypt. ${ }^{3}$ Department of Pharmacology and Toxicology, College of Pharmacy, King Saud University, P.O. Box 2457, Riyadh 11451, Saudi Arabia. ${ }^{4}$ Cancer biology Department, Virology and Immunology Unit, National 
Cancer Institute, Cairo University, Cairo, Egypt. ${ }^{5}$ Department of Infection and Immunity, King Faisal Specialist Hospital and Research Centre, Riyadh, Saudi Arabia. ${ }^{6}$ College of Medicine, Alfaisal University, Riyadh, Saudi Arabia. ${ }^{7}$ Preventive Medicine Directorate, Ministry of Health, Riyadh, Saudi Arabia.

${ }^{8}$ Microbiology section, Prince Salman Hospital, Riyadh, Saudi Arabia.

Received: 11 September 2013 Accepted: 1 January 2014

Published: 7 January 2014

\section{References}

1. Pitout JD, Laupland KB: Extended-spectrum $\beta$-lactamase-producing Enterobacteriaceae: an emerging public-health concern. Lancet Infect Dis 2008, 8(3):159-166

2. Canto'n R, Coque TM: The CTX-M beta-lactamase pandemic. Curr Opin Microbiol 2006, 9:466-475.

3. Peirano G, Pitout JDD: Molecular epidemiology of Escherichia coli producing CTX-M $\beta$-lactamases: the worldwide emergence of clone ST131 O25:H4. Int J Antimicrob Agents 2010, 35:316-321.

4. Banerjee R, Johnson JR: Escherichia coli ST131: Variations on a theme of clonal expansion. Enferm Infecc Microbiol Clin 2013, 31(6):355-356.

5. Banerjee R, Johnston B, Lohse C, Porter SB, Clabots C, Johnson JR: Escherichia coli sequence type 131 is a dominant, antimicrobial-resistant clonal group associated with healthcare and elderly hosts. Infect Control Hosp Epidemiol 2013, 34(4):361-369.

6. Dhanji H, Doumith M, Clermont O, Denamur E, Hope R, Livermore DM, et al: Real-time PCR for detection of the O25b-ST131 clone of Escherichia coli and its CTX-M-15-like extended-spectrum beta-lactamases. Int J Antimicrob Agents 2010, 36(4):355-358.

7. Rogers BA, Sidjabat HE, Paterson DL: Escherichia coli O25b-ST131: a pandemic, multiresistant, community-associated strain. J Antimicrob Chemother 2011, 66(1):1-14.

8. Vimont S, Boyd A, Bleibtreu A, Bens M, Goujon J-M, Garry L, Clermont O, Denamur E, Arlet G, Vandewalle A: The CTX-M-15-producing Escherichia coli clone O25b: H4-ST131 has high intestine colonization and urinary tract infection abilities. PLoS One 2012, 7(9):e46547.

9. Al-Agamy MH, Shibl AM, Elkhizzi NA, Meunier D, Turton JF, Livermore DM: Persistence of Klebsiella pneumoniae clones with OXA-48 or NDM carbapenemases causing bacteraemias in a Riyadh hospital. Diagn Microbiol Infect Dis 2013, 76(2):214-216.

10. Al-Agamy MH, Shibl AM, Tawfik AF: Prevalence and molecular characterization of extended-spectrum $\beta$-lactamase-producing Klebsiella pneumoniae in Riyadh, Saudi Arabia. Ann Saudi Med 2009, 29(4):253-257.

11. Ahmad S, Al-Juaid NF, Alenzi FQ, Mattar EH, Bakheet O-S: Prevalence, antibiotic susceptibility pattern and production of extended-spectrum betalactamases amongst clinical isolates of Klebsiella pneumoniae at armed forces hospital in Saudi Arabia. J Coll Phys Surg Pak 2009, 9(4):264-265.

12. Shibl AM, Al-Agamy MH, Khubnani H, Senok AC, Tawfik AF, Livermore DM: High prevalence of acquired quinolone-resistance genes among Enterobacteriaceae from Saudi Arabia with CTX-M-15 $\beta$-lactamase. Diagn Microbiol Infect Dis 2012, 73(4):350-353.

13. Tawfik AF, Alswailem AM, Shibl AM, Al-Agamy MH: Prevalence and genetic characteristics of TEM, SHV, and CTX-M in clinical Klebsiella pneumoniae isolates from Saudi Arabia. Micro Drug Resist 2011, 17(3):383-388.

14. Kader AA, Kumar A: Prevalence and antimicrobial susceptibility of extendedspectrum beta-lactamase-producing Escherichia coli and Klebsiella pneumoniae in a general hospital. Ann Saudi Med 2005, 25(3):239-242.

15. Khanfar HS, Bindayna KM, Senok AC, Botta GA: Extended spectrum betalactamases (ESBL) in Escherichia coli and Klebsiella pneumoniae: trends in the hospital and community settings. J Infect Dev Ctries 2009, 3(4):295-299.

16. Al-Otaibi FE, Bukhari EE: Clinical and laboratory profiles of urinary tract infections caused by extended-spectrum beta-lactamase-producing Escherichia coli in a tertiary care center in central Saudi Arabia. Saudi Med J 2013, 34(2):171-176.

17. Bindayna K, Khanfar HS, Senok AC, Botta GA: Predominance of CTX-M genotype among extended spectrum beta lactamase isolates in a tertiary hospital in Saudi Arabia. Saudi Med J 2010, 31(8):859-863.

18. Clinical and Laboratory Standards Institute: Methods for dilution antimicrobial susceptibility tests for bacteria that grow aerobically, $7^{\text {th }}$ ed. Approved standard M02-A11. Wayne: CLSl; 2012.

19. Dallenne C, Da Costa A, Decré D, Favier C, Arlet G: Development of a set of multiplex PCR assays for the detection of genes encoding important betalactamases in Enterobacteriaceae. J Antimicrob Chemother 2010, 65:490-495.
20. Woodford N, Ward ME, Kaufmann ME, Turton J, Fagan EJ, James D, Johnson AP, Pike R, Warner M, Cheasty T, Pearson A, Harry S, Leach JB, Loughrey A, Lowes JA, Warren RE, Livermore DM: Community and hospital spread of Escherichia coli producing CTX-M extended-spectrum $\beta$-lactamases in the UK. J Antimicrob Chemother 2004, 54(4):735-743.

21. Gautom RK: Rapid pulsed-field gel electrophoresis protocol for typing of Escherichia coli 0157:H7 and other gram-negative organisms in 1 day. J Clin Microbiol 1997, 35(11):2977-2980.

22. Tenover FC, Arbeit RD, Goering RV, Mickelsen PA, Murray BE, Persing DH, Swaminathan B: Interpreting chromosomal DNA restriction patterns produced by pulsed-field gel electrophoresis: criteria for bacterial strain typing. J Clin Microbiol 1995, 33(9):2233-2239.

23. Bonnet R, Recule C, Baraduc R, Chanal C, Sirot D, De Champs C, Sirot Effect of D240G substitution in a novel ESBL CTX-M-27. J Antimicrob Chemother 2003, 52(1):29-35.

24. Nicolas-Chanoine MH, Blanco J, Leflon-Guibout V, Demarty R, Alonso MP, Canica MM, Park YJ, Lavigne JP, Pitout J, Johnson JR: Intercontinental emergence of Escherichia coli clone O25:H4-ST131 producing CTX-M-15. J Antimicrob Chemother 2008, 61(2):273-281.

25. Lau SH, Reddy S, Cheesbrough J, Bolton FJ, Willshaw G, Cheasty T, Fox AJ, Upton M: Major uropathogenic Escherichia coli strain isolated in the northwest of England identified by multilocus sequence typing. J Clin Microbiol 2008, 46(3):1076-1080

26. Johnson JR, Johnston B, Clabots C, Kuskowski MA, Castanheira M: Escherichia coli sequence type ST131 as the major cause of serious multidrug-resistant E. coli infections in the United States. Clin Infect Dis 2010, 51(3):286-294.

27. Sidjabat HE, Paterson DL, Adams-Haduch JM, Ewan L, Pasculle AW, Muto CA, Tian GB, Doi Y: Molecular epidemiology of CTX-M-producing Escherichia coli isolates at a tertiary medical center in western Pennsylvania. Antimicrob Agents Chemother 2009, 53:4733-4739.

28. Severin JA, Mertaniasih NM, Kuntaman K, Lestari ES, Purwanta M, Lemmens-Den Toom N, Duerink DO, Hadi U, van Belkum A, Verbrugh HA, Goessens WH, Study Group 'Antimicrobial Resistance in Indonesia: Prevalence and Prevention' (AMRIN): Molecular characterization of extended-spectrum beta-lactamases in clinical Escherichia coli and Klebsiella pneumoniae isolates from Surabaya, Indonesia. J Antimicrob Chemother 2010, 65(3):465-469.

29. Markovska R, Schneider I, Ivanova D, Keuleyan E, Stoeva T, Sredkova M, Markova B, Bojkova K, Gergova R, Bauernfeind A, Mitov I: High prevalence of CTX-M-15-producing O25b-ST131 Escherichia coli clone in Bulgarian hospitals. Microb Drug Resist 2012, 18(4):390-395.

30. Pitout JD, Gregson DB, Campbell L, Laupland KB: Molecular characteristics of extended-spectrum- $\beta$-lactamase-producing Escherichia coli isolates causing bacteremia in the Calgary health region from 2000 to 2007: emergence of clone ST131 as a cause of community-acquired infections. Antimicrob Agents Chemother 2009, 53(7):2846-2851.

31. Smet A, Martel A, Persoons D, Dewulf J, Heyndrickx M, Claeys G, Lontie M, Van Meensel B, Herman L, Haesebrouck F, Butaye P: Characterization of extended-spectrum $\beta$-lactamases produced by Escherichia coli isolated from hospitalized and nonhospitalized patients: emergence of CTX-M15-producing strains causing urinary tract infections. Microb Drug Resist 2010, 16(2):129-134.

32. Peirano G, Costello M, Pitout JD: Molecular epidemiology over an 11-year period (2000 to 2010) of extended-spectrum $\beta$-lactamase- producing Escherichia coli causing bacteremia in a centralized Canadian region. Int $\lrcorner$ Antimicrob Agents 2010, 36(1):19-23.

33. Croxall G, Hale J, Weston V, Manning G, Cheetham P, Achtman M, McNally A: Molecular epidemiology of extraintestinal pathogenic Escherichia coli isolates from a regional cohort of elderly patients highlights the prevalence of ST131 strains with increased antimicrobial resistance in both community and hospital care settings. J Antimicrob Chemother 2011, 66(11):2501-2508.

34. Peirano G, van der Bij AK, Gregson DB, Pitout JD: Molecular epidemiology over an 11-year period (2000 to 2010) of extended-spectrum $\beta$ lactamase-producing Escherichia coli causing bacteremia in a centralized Canadian Region. J Clin Microbiol 2012, 50(2):294-299.

doi:10.1186/1476-0711-13-4

Cite this article as: Al-Agamy et al:: Molecular characteristics of extended-spectrum $\beta$-lactamase-producing Escherichia coli in Riyadh: emergence of CTX-M-15-producing E. coli ST131. Annals of Clinical Microbiology and Antimicrobials 2014 13:4 